\title{
IPSI vs CONTRA TRAM—Old Doubts New Answers: Evaluation TRAM Flap Survival with Static and Active Dynamic Thermography in Pedicled Flap Breast Reconstruction
}

\author{
Jerzy Jankau ${ }^{1}$ • Szymon Kolacz ${ }^{2}$ - Mateusz Moderhak ${ }^{3}$ \\ Received: 1 August 2019 / Accepted: 11 March 2020 / Published online: 1 April 2020 \\ (C) The Author(s) 2020
}

\begin{abstract}
Breast reconstruction is increasingly being considered as the next step in breast cancer treatment. There are many ways to recreate a new breast mound. In current times, the trend for breast reconstruction is reconstruction microsurgery which is the method of choice, but newcomers should know the alternative traditional methods. Since 1982 the pedicled TRAM (trans rectus abdominal myocutaneous) flap has become one of the most popular autologous tissue breast reconstruction procedures. It has also been debated for many years which method of transferring the tissue island is more reliable and has fewer complications--IPSI vs CONTRA [1]. The reasons for complications are the manner of twisting the nourishing blood supply and the tension due to the distance of the pedicle in both procedures [2]. Static thermography and dynamic thermography seem to provide the solution. In our experiment, we measured the medial surface temperature of flaps using static thermography and active dynamic thermography. The results were compared against the number of flap failures in each type of reconstruction. The study was conducted on a group of 20 female patients who underwent breast reconstruction.
\end{abstract}

Keywords Breast reconstruction · Thermography $\cdot$ Active dynamic thermography $\cdot$ TRAM flap $\cdot$ Flap survival $\cdot$ Skin necrosis · Flap necrosis

\section{Introduction}

Among the most common plastic surgery methods used in breast reconstructions are pedicled flaps. One of the examples is the skin-muscle TRAM (trans rectus abdominal myocutaneous flap) flap. Despite the fact that its anatomy is well-explored [3], its partial necrosis may develop as a consequence of twisting and tension on the nourishing blood

We declare the approval of an Independent Bioethics Committee No. 590/ 2013 in Gdansk Poland for the project entitled: "Perfusion imaging of tissue flaps in real time using Active Thermography."

Jerzy Jankau

jjankau@gumed.edu.pl

1 Department of Plastic Surgery, Medical University of Gdansk, Smoluchowskiego 17, 80-214 Gdansk, Poland

2 Department of Plastic Surgery, Dubai London Clinic and Hospital, Jumeirah Beach road, Umm Suqeim 2, P.O.Box 12119, Dubai, UAE

3 Departament of Biomedical Engineering, Gdansk University of Technology, 11/12 G. Narutowicza St., 80-233 Gdańsk, Poland supply. In order to minimalize the risk of flap failure, some surgeons decide to use IPSI-lateral flap which involves less tension but more flap pedicle rotation. Other surgeons, however, use the CONTRA-lateral flap with more tension but less rotation of flap pedicle. Hartrampf's original paper presents the use of TRAM IPSI-lateral flap [4].

Nowadays both CONTRA-lateral and IPSI-lateral flaps are used, and the choice depends on the surgeon's experience and the patient's anatomy. Available literature refers to the reports on the impact of tension and rotation of the vascular pedicle on the flap blood supply, suggesting more favourable conditions provided by IPSI-lateral flaps [2]. It is, however, impossible to overlook the reports on the lack of significant differences between IPSI-lateral and CONTRA-lateral flaps [5]. From a plastic surgeon's viewpoint, establishing which flap has better blood supply is critical. It contributes to adequate planning of a surgical procedure and thus reducing the rate of potential complications such as partial flap necrosis, prolonged hospitalization, number of reoperations, or healthcare service cost while offering better aesthetic results and patient satisfaction (Figs. 1 and 2).

Adequate preoperative planning is very important in reconstructive surgery. In order to minimalize the risk of flap failure, 


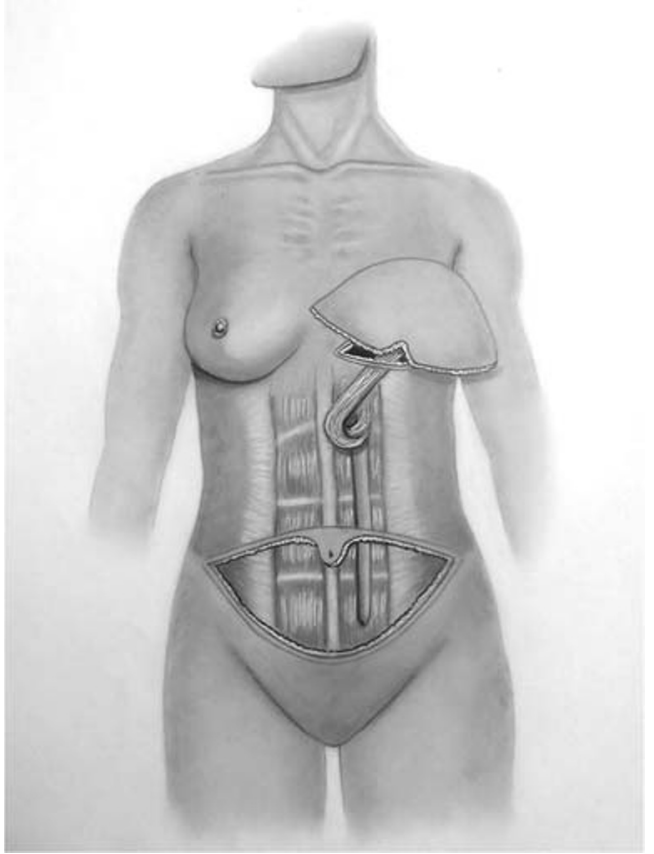

Fig. 1 IPSI-lateral TRAM flap

it is essential to choose the flap with better blood perfusion. The aim of our research was to compare the clinical examination of TRAM flap and the images generated in intraoperative static thermography and cold-stress active dynamic thermography (ADT) both prior to and after twisting the vascular pedicle of the flap, and in subsequent postoperative days in the reconstructive procedures with TRAM IPSI-lateral and

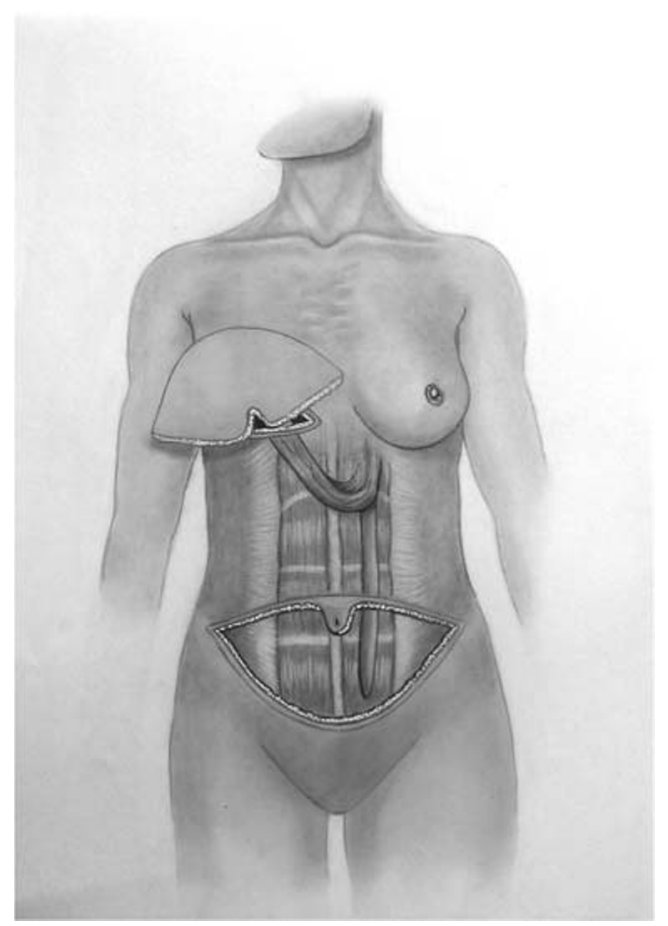

Fig. 2 CONTRA-lateral TRAM flap
CONTRA-lateral flaps. It is well known that there is a specific relationship between skin blood perfusion and temperature so we decided to examine it with a static thermograph device [6] and cold-stress active dynamic thermography [7].

\section{Material and Methods}

The study involved a group of 20 patients undergoing TRAM flap breast reconstruction. Half of them underwent IPSI-lateral breast reconstruction, and the other half underwent CONTRAlateral breast reconstruction. All treatments were provided by the same operating team, in the same operating room with constant temperature. The variables found in the study group include the following number of smokers in the last 90 days was 2 in the CONTRA-lateral group. Five patients from the IPSI-lateral group and 4 patients from the CONTRA-lateral group underwent chemotherapy. One IPSI-lateral patient and 2 CONTRA-lateral patients underwent radiotherapy.

The first imaging examination was performed before the surgery (b1), the second one after the dissection of the skin-muscle flap and after excision of its excesses (b2), the third one immediately after suturing flap in the recipient region (b3), and the follow-up ones at 1 (b4), 7 (b5), 30 (b6), and 90 (b7) days after the surgery. Each examination consisted of the following steps:

1. Static thermal imaging.

2. Dynamic thermal imaging with high energy stimulation of the flap for $60 \mathrm{~s}$ and monitoring changes in temperature over the next $180 \mathrm{~s}$. A set of 20 halogen lamps (1000 W) provided stimulation.

3. Dynamic thermal imaging with low energy stimulation of the flap for $60 \mathrm{~s}$ and monitoring changes in temperature over the next $180 \mathrm{~s}$.

The collected data were then processed to yield results in a numerical form, and the precise description of the study method is already published [8].

All patients were operated by the same team in general anaesthesia. Each operation began with umbilicus dissection and flap elevation. The skin was incised along the entire length of the skin-fat flap from the left anterior superior iliac spine to the right one. The delimiting lower line was $7 \mathrm{~cm}$ from the superior angle of labial commissure, and the superior line was at the level of navel. The skin-fat flap was raised from the side medially until reaching the lateral border of rectus abdominal muscle. The anterior sheath was incised to uncover inferior epigastric vessels which were ligated and cut along with lower border of the rectus muscle. In upward direction, the skin-flap was lifted up to the border of xiphoid and costal arch. The anterior sheath was incised along the rectus muscle to enable lifting the skin-muscle TRAM flap. To protect against the excessive extension of the pedicle, a 3-cm-wide fascia was left. 


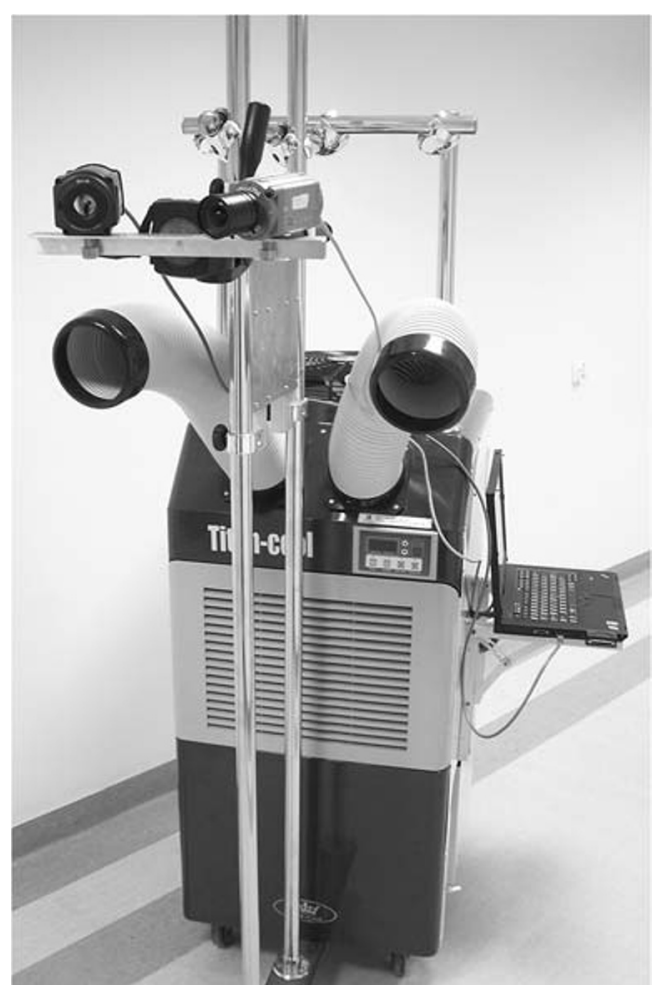

Fig. 3 Active thermographic station

The dissected TRAM flap was at this point modelled by means of dissecting the entire zone IV, with a part of zone II and III.

The next stage was preparing the recipient region. Started with excision of the amputation cicatrix, lifting the dermal subcutaneous flaps and followed by dissecting a subcutaneous tunnel in the region of the xiphoid and costal arch, what allowed to transfer the flap and to form a new breast mound.

At the final stage of the preparation, careful haemostasis was performed, and the umbilicus was elevated and sutured with Vicryl 4-0 and 5-0 nylon. The wounds were closed with Vicryl 2-0 and 4-0 sutures and V-Loc 4-0 wound closure device, respectively (Fig. 3).

For the purposes of this work, the static and cold stress dynamic thermography results were collected from intraoperative studies directly after rotating and subsequent inserting the flap into the recipient site (b3), on postoperative days 1 (b4) and 7 (b5). The results of the preoperative (b1) exam and intraoperative

Table 1 Results of static thermography on subsequent days in IPSI flaps

\begin{tabular}{llll}
\hline IPSI & $\mathrm{b}-3$ & $\mathrm{~b}-4$ & $\mathrm{~b}-5$ \\
\hline Mean temp. & & & \\
Mean (SD) & $302.4(1.5)$ & $305.8(1.6)$ & $305.7(1.2)$ \\
95\% CI & {$[301.3 ; 303.5]$} & {$[304.4 ; 307.2]$} & {$[304.7 ; 306.7]$} \\
Range (min-max) & $300.4-304.5$ & $304.0-308.1$ & $304.0-308.1$ \\
Median & 302.4 & 305.9 & 305.5 \\
\hline
\end{tabular}

Table 2 Results of static thermography on subsequent days in CONTRA flaps

\begin{tabular}{llll}
\hline CONTRA & $\mathrm{b}-3$ & $\mathrm{~b}-4$ & $\mathrm{~b}-5$ \\
\hline Mean temp. & & & \\
Mean (SD) & $301.7(2.5)$ & $307.0(1.0)$ & $307.5(1.2)$ \\
$95 \%$ CI & {$[299.9 ; 303.6]$} & {$[306.3 ; 307.8]$} & {$[306.7 ; 308.3]$} \\
Range (min-max) & $298.6-306.1$ & $305.7-308.8$ & $306.2-310.3$ \\
Median & 301.1 & 307.1 & 307.6 \\
\hline
\end{tabular}

exam performed following the dissection and resection of excess flap (b2), and the exam performed after 30 and 90 days (b6 and b7) were not included in this study because of the lack of relevance. The results and the number of flat failure cases were compared between groups. During the entire study period, there were no cases of complete flap necrosis, which would indicate that the pedicle was compressed or cut (Tables 1, 2, 3, 4 and 5).

\section{Results}

The answer to the question whether IPSI-lateral or CONTRAlateral flaps have better blood supply should be sought after TRAM displacement starting at intraoperative b3 when flap is already rotated and advanced.

In the case of static thermography, the average mean and median temperature in the intraoperative b3 examination is higher for the IPSI-lateral flaps, and it is the higher in the CONTRA-lateral flaps in the first and the seventh days postoperatively. Interpreting these results involves considering the nature of static thermography which measures surface temperature at a given moment. Given that the normal human temperature is within the range of $35.5-37^{\circ} \mathrm{C}$ [9], we cannot say that people whose temperature is nearer to $37^{\circ} \mathrm{C}$ are healthier than those whose results are closer to $36^{\circ} \mathrm{C}$ because they are all within the scope of the standard values. Therefore, the direct comparison of static thermography in IPSI-lateral and CONTRA-lateral is not objectively relevant. It is more important to observe the increase in mean and median temperature values in flaps in the following days, which is evidence of progressive stabilization of circulation in the flap.

Table 3 Results of dynamic cooling thermography on subsequent days in IPSI flaps

\begin{tabular}{llll}
\hline & $b-3$ & $b-4$ & $b-5$ \\
\hline dTnorm sr & & & \\
Mean (SD) & $0.49(0.10)$ & $0.55(0.07)$ & $0.58(0.10)$ \\
$95 \%$ CI & {$[0.40 ; 0.58]$} & {$[0.49 ; 0.61]$} & {$[0.50 ; 0.65]$} \\
Range (min-max) & $0.29-0.58$ & $0.46-0.67$ & $0.48-0.76$ \\
Median & 0.51 & 0.56 & 0.56 \\
\hline
\end{tabular}


Table 4 Results of dynamic cooling thermography on subsequent days in CONTRA flaps

\begin{tabular}{lccc}
\hline & b-3 & b-4 & b-5 \\
\hline dTnorm sr & & & \\
Mean (SD) & $0.45(0.10)$ & $0.53(0.05)$ & $0.55(0.06)$ \\
$95 \%$ CI & {$[0.35 ; 0.56]$} & {$[0.48 ; 0.58]$} & {$[0.51 ; 0.60]$} \\
Range (min-max) & $0.27-0.54$ & $0.47-0.60$ & $0.48-0.66$ \\
Median & 0.48 & 0.54 & 0.54 \\
\hline
\end{tabular}

Cold stress dynamic thermography is apparently in opposition to static thermography. It measures the speed of temperature changes along a 180 -s-long period following $60 \mathrm{~s}$ of cooling, which leads to indicating the quality of flap blood supply. In the b3 study, i.e. immediately after suturing the flap in the recipient site, the mean dTnorm and median values are higher in the IPSI-lateral flaps than in the CONTRA-lateral flaps. The same results are obtained on the first and seventh day postoperatively (Figs. 4 and 5).

The study also involved clinical evaluation with photographic documentation. The number of marginal TRAM flap necrosis complications was higher in IPSI flaps than in CONTRA flaps. However, the total area of marginal necrosis was about $10 \%$ of the flap area in the IPSI-lateral flaps and $2.5 \%$ of the total flap area in CONTRA-lateral flaps. In spite of the larger surface area of IPSI-lateral flap necrosis, IPSI-lateral flaps still had higher dTnorm values in dynamic thermography than CONTRAlateral flaps, which in turn was evidence of better blood supply. Despite the fact that the anatomy of TRAM flaps [4] is wellknown, marginal flap necrosis may occur due to the lack of welldefined borders between the blood supply zones (Fig. 6).

\section{Discussion}

The ideal diagnostic tool for skin flap circulation monitoring was characterized by Creech, B. and Miller, S. as early as 1975 [10]:

- Reproducible and non-invasive

- Safe for the patient

- Safe for the flap

- Cost-effective in terms of purchase and exploitation

There are many methods that illustrate the flap blood supply which can be used to evaluate TRAM IPSI-lateral and CONTRA-lateral flap. These include methods such as laser

Table 5 Total number of reconstructions with the number of complications in particular groups

\begin{tabular}{lll}
\hline & IPSI & CONTRA \\
\hline Total number & 10 & 10 \\
Partial flap necrosis & 4 & 1 \\
\hline
\end{tabular}

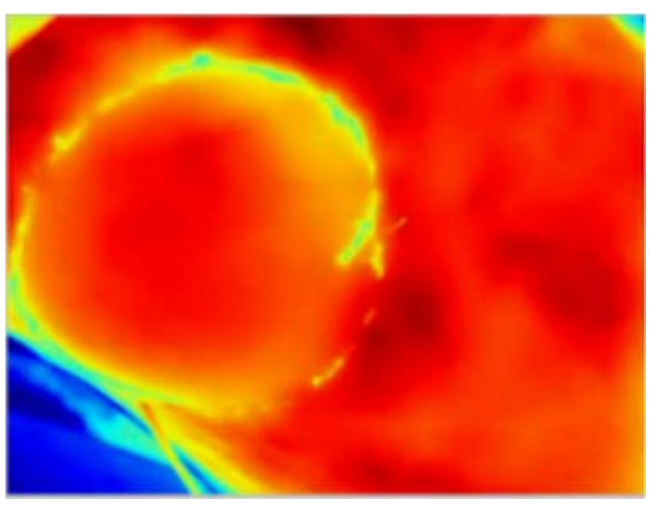

Fig. 4 Samples of static thermography

Doppler, thermography, indocyanine green, angiography and angio-MRI. Angiography, angio-MRI and indocyanine green are highly sensitive studies. However, they are invasive, which may cause allergic reactions in patients and are difficult to do at the patient's bedside, and frequent repetitions are often required as needed.

Laser Doppler and thermography methods are the closest to fulfil the conditions of ideal monitoring tool. They are non-invasive, low cost, reliable and repeatable and have the possibility to be performed at the patient's bedside and in the operating room. The only and biggest disadvantage of the laser Doppler is its high price, which we cannot say about a thermal imaging camera.

With this in mind, we decided to use active dynamic thermography to assess the blood supply to the TRAM IPSIlateral and CONTRA-lateral flaps.

It is estimated that around $60-70 \%$ of women suffering from breast cancer undergo breast-conserving therapy (BCT) without having their entire breast removed [11]. Bearing in mind the self-awareness of the patients, breast reconstruction surgery is becoming increasingly frequent. For small defects, the use of local flaps or autologous adipose tissue transplantation may be a good way to reconstruct the breast. However, in the case of large or unfavourably situated tumours, the patient should be informed about the possible unsatisfactory appearance of the breast after the procedure. Another situation is the appearance of the breast reconstructed with implant and subsequent

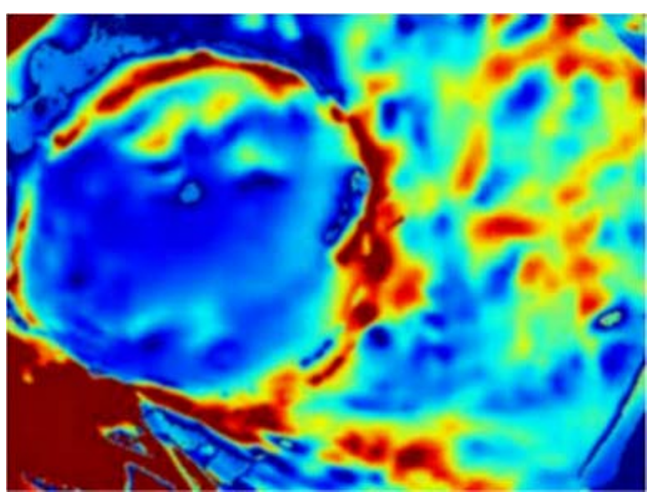

Fig. 5 Samples of active dynamic thermography with cooling 


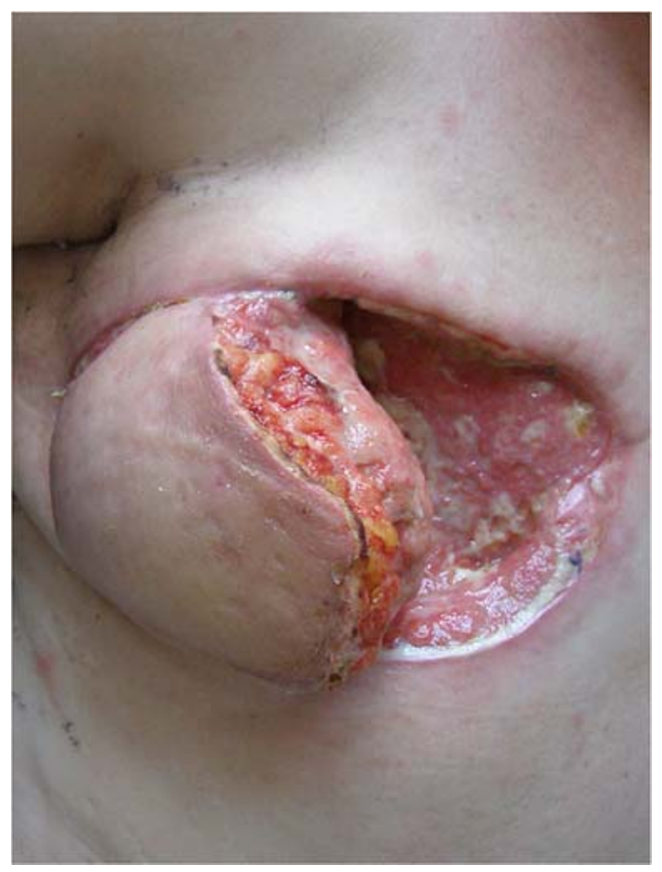

Fig. 6 Sample of TRAM flap after debridement of marginal necrosis

radiation therapy. Due to the excessive capsular contraction and rippling, the breasts may look unsatisfactory. In these situations, secondary surgery often involves the use of autologous tissues. Depending on the patient's preferences, her anatomical structures, hospital equipment and microsurgical skills of the surgical team, a free DIEP (deep inferior epigastric perforator) flap may be used or, as an alternative, a pedicled TRAM flap.

The pedicled TRAM flap will fulfil its role especially in overweight patients and in whom, for various reasons, prolonged surgery is contraindicated.

Therefore, the answer to the question which TRAMIPSI-lateral or CONTRA-lateral flap has better blood supply is still there.

Unfortunately, due to scarce literature on the subject, it is impossible to make relevant references. Clugston's research [2] found that lower venous pressure due to vascular rotation and vasoconstriction was maintained in IPSI flaps, which is in line with our findings.

\section{Conclusions}

Considering our findings, we found that IPSI-lateral TRAM flaps have better blood supply than CONTRA-lateral TRAM flaps. However, it should be noted that each surgeon should take his or her own experience and expertise into consideration while selecting the method which is appropriate for a particular patient and can offer the best aesthetic effect.

Author Contributions Study conception and design: Szymon Kolacz, Mateusz Moderhak, Jerzy Jankau.

Acquisition of data: Jerzy Jankau, Szymon Kolacz.
Analysis and interpretation of data: Szymon Kolacz, Mateusz Moderhak.

Drafting of manuscript: Jerzy Jankau.

Critical revision: Jerzy Jankau, Szymon Kolacz, Mateusz Moderhak.

\section{Compliance with Ethical Standards}

Conflict of Interest The authors declare that they have no conflict of interest.

Open Access This article is licensed under a Creative Commons Attribution 4.0 International License, which permits use, sharing, adaptation, distribution and reproduction in any medium or format, as long as you give appropriate credit to the original author(s) and the source, provide a link to the Creative Commons licence, and indicate if changes were made. The images or other third party material in this article are included in the article's Creative Commons licence, unless indicated otherwise in a credit line to the material. If material is not included in the article's Creative Commons licence and your intended use is not permitted by statutory regulation or exceeds the permitted use, you will need to obtain permission directly from the copyright holder. To view a copy of this licence, visit http://creativecommons.org/licenses/by/4.0/.

\section{References}

1. Clugston PA, Gingrass MK, Azurin D, Fisher J, Maxwell GP (2000) Ipsilateral pedicled TRAM flaps: the safer alternative? Plast Reconstr Surg 105(1):77-82

2. Clugston PA, Lennox PA, Thompson RP (1998) Intraoperative vascular monitoring of ipsilateral vs. contralateral TRAM flaps. Ann Plast Surg 41(6):623-628

3. Moon HK, Taylor GI (1998) The vascular anatomy of rectus abdominis musculocutaneous flaps based on the deep superior epigastric system. Plast Reconstr Surg 82(5):815-32

4. Hartrampf CR Jr, Scheflan M, Black PW (1982) Breast reconstruction with a transverse abdominal island flap. Plast Reconstr Surg 69:216-225

5. Janiga TA, Atisha DM, Lytle IF, Wilkins EG, Alderman AK (2010) Ipsilateral pedicle TRAM flaps for breast reconstruction: are they as safe as contralateral techniques? J Plast Reconstr Aesthet Surg 63:322-326

6. Francis JE, Roggli RI, Love TJ (1979) Thermography as means of blood perfusion measurement. J Biomech Eng 101:246-251

7. Sz K, Moderhak M, Jankau J (2017) New perspective on the in vivo use of cold stress dynamic thermography in integumental reconstruction with the use of skin-muscle flaps. J Surg Res 212:68-76

8. Moderhak M, Kołacz S, Jankau J, Juchniewicz T (2017) Active dynamic thermography method for TRAM flap blood perfusion mapping in breast reconstruction. QIRT J 14:1-16 Published online: 04. https://doi.org/10.1080/17686733.2017.1320885

9. Sund-Levander M, Forsberg C, Wahren LK (2002) Normal oral, rectal, tympanic and axillary body temperature in adult men and women: a systematic literature review. Scand J Caring Sci 16(2):122-128

10. Creech B, Miller S (1975) Evaluation of circulation in skin flaps. In: Grabb WC, Myers MB (eds) Skin flaps. Little, Brown, Boston

11. Munder B, Seidenstuecker K, Richrath P, Behrendt P, Köppe T, Hagouan M, Audretsch W, Nestle-Krämling C, Witzel C (2012) Skin-sparing mastectomy and immediate reconstruction with DIEP flap after breast-conserving therapy. Med Sci Monit 18(12):CR716-CR720

Publisher's Note Springer Nature remains neutral with regard to jurisdictional claims in published maps and institutional affiliations. 